\section{Is rehabilitation post critical illness a new anti-inflammatory agent?}

\author{
Doug Elliott, ${ }^{1}$ Zudin Puthucheary ${ }^{2}$
}

Physical recovery following a critical illness is important for patients, family and clinicians. $^{1-3}$ However, there is limited beneficial effect demonstrated from the intervention studies that have investigated physical rehabilitation across the continuum of critical illness recovery, starting in the intensive care unit (ICU) and continuing to the community setting with home-based rehabilitation and follow-up clinics $^{4-8}$ What is the reason for this lack of treatment effect? As with all trials that show a lack of effect, we must consider a number of factors, including the intervention delivered, the target population and the outcome measure used.

In the recent RECOVER trial, ${ }^{8}$ there was an improvement in the functional status of patients following critical illness, as evidenced by the Rivermead Mobility Index (RMI); however, the delivery of a post-ICU bundled therapies intervention did not enhance the physical outcome over and above the normal trajectory of recovery. As there was clear demonstration that the treatment was delivered, this raises the question of whether the target population was appropriate and, in particular, whether we can identify cohorts of patients that are more or less likely to respond to rehabilitation treatment.

In Thorax, Griffith et $a l^{9}$ report an a priori secondary analysis of the RECOVER trial. ${ }^{8}$ In this substudy, 193 representative patients were included. The key observation was that a pro-inflammatory state persisted up to 3 months post discharge and that hydroxyonenal and $\mathrm{C}$ reactive protein (CRP) were independently associated with persistent limitations at 3 months post-ICU discharge for mobility, as measured by the RMI, and muscle strength, as measured by handgrip strength. Furthermore, previous exposure to human cytomegalovirus (CMV) was associated with a pro-inflammatory phenotype of persistent inflammation that may influence functional recovery. ${ }^{9}$ The

\footnotetext{
${ }^{1}$ Faculty of Health, University of Technology Sydney, Sydney, New South Wales, Australia; ${ }^{2}$ Institute of Health and Human Performance, University College London, London, UK
}

Correspondence to Dr Doug Elliott, Faculty of Health, University of Technology Sydney, 15 Broadway, Ultimo, NSW 2007, Australia; Doug.Elliott@uts.edu.au authors acknowledge that the measurement of muscle recovery was limited to functional assessment only and there were no structural or biological measures undertaken, which limits full interpretation of the observation. As with all observational studies, a causal link between persistent systemic inflammation and physical function outcome can only be postulated.

What can we postulate from these findings? Although the potential stratification of patients may be possible in terms of persistent systemic inflammation as almost a third of the observational cohort demonstrated a CRP value $>10 \mathrm{mg} / \mathrm{L}$, the relationship between systemic inflammation functional ability at 3 months was inconsistent, being different for the objective and subjective assessment of physical function. While associations were demonstrated between systemic inflammation and the RMI and handgrip strength, there was no relationship with the physical component score of short-form 12. The reasons for these divergent findings remain unclear, and this will need further investigation.

The current study ${ }^{9}$ highlights the importance that persistent systematic inflammation has on physical recovery and supports a recent systematic review ${ }^{10} \mathrm{dem}$ onstrating systemic inflammation and the systematic inflammatory response syndrome in patients at ICU discharge. It is perhaps not surprising that there is a focus on the area of persistent inflammation in terms of muscle biology and function as it is known that inflammation depresses muscle protein synthesis in both human ${ }^{11} 12$ and animal models. ${ }^{13}$ This inflammatory process results in impairment of the muscle protein synthetic response to exercise and nutrition. Complex interactions between systemic and local inflammation and age may further influence muscle protein homeostasis with older patients likely to have age-related anabolic resistance. ${ }^{14}$ In addition, systemic inflammation may exacerbate the observed fasciitis $^{15}$ and myonecrosis, ${ }^{15}$ impairing muscle repair ${ }^{16}$ and functional outcome. ${ }^{17}$

However, the actual picture may not be so clear for individuals exposed to a critical illness. Raised interleukin (IL)-6 levels increase fatty acid oxidation (increasing ATP production) and stimulate translocation of the glucose transporter protein GLUT-4 to the plasma membrane. ${ }^{18}$ In this setting, we may be witnessing metabolic recovery from the physiological derangements of critical illness ${ }^{19} \quad 20$ and a raised IL-8 perhaps marking autophagy of damaged necrotic tissue, ${ }^{21}$ suggesting that there may be an appropriate rehabilitation window. ${ }^{22}$

In addition to the observation of persistent systemic inflammation, previous exposure to CMV was associated with a pro-inflammatory state. CMV activation can shift macrophage function from antiinflammatory to pro-inflammatory, ${ }^{23}$ preventing recovery from muscle necrosis and damage that occurs in up to $40 \%$ of patients. This is supported by lower concentrations of the anti-inflammatory cytokine transforming growth factor beta 1 (TGF- $\beta 1$ ) in those with previous CMV exposure. The potential role of CMV in preventing effective rehabilitation has biological plausibility, but the demonstration of CMV activation and the mechanism through which CMV infection inhibits the generation of TGF- $\beta 1$ would strengthen these findings considerably.

Patient heterogeneity therefore needs to be carefully considered as this will influence the inflammatory profile and trajectory of recovery. Patients with and without pre-existing chronic disease states have different recovery profiles in terms of physical activity $^{24}$ and therefore controlling confounders such as interindividual variation is essential in complex rehabilitation interventional trials. ${ }^{25}$ Our current understanding of patient heterogeneity and the process of stratification has been facilitated by the reporting of functional recovery and survival trajectories in patients who received invasive mechanical ventilation for $>7$ days. $^{26}$ Although the data from Griffith and colleagues indicate that the inflammatory profile is unrelated to either comorbidity or function, ${ }^{9}$ these data highlight that inflammatory biomarkers, as well as age and comorbidity, need to be considered for stratification of the patients who are likely to benefit from rehabilitation. Previous data would support the concept that the severity of the acute illness determines the degree of skeletal muscle wasting ${ }^{21}$ and patient comorbidity influences the trajectory of recovery, ${ }^{24}$ but now we must consider the mechanism through which persistent systemic inflammation plays a key role in outcome.

These data strongly support the requirement for biological science to provide the rationale for stratification of rehabilitation treatment interventions, and perhaps we should consider that rehabilitation be targeted as an anti-inflammatory treatment 
in those patients with persistent systemic inflammation post critical illness.

Competing interests None declared.

Provenance and peer review Commissioned; externally peer reviewed.

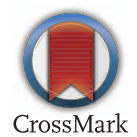

To cite Elliott $D$, Puthucheary $Z$. Thorax 2016;71:783-784.

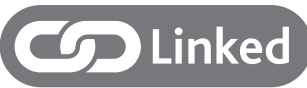

http://dx.doi.org/10.1136/thoraxjnl-2015-208114

Thorax 2016:71:783-784.

doi:10.1136/thoraxjnl-2016-208844

\section{REFERENCES}

1 Castro-Avila $A C$, Serón $P$, Fan $E$, et al. Effect of early rehabilitation during Intensive Care Unit Stay on Functional Status: Systematic Review and Meta-Analysis. PLOS ONE 2015;10:e130722.

2 Rydingsward JE, Horkan CM, Mogensen KM, et al. Functional status in ICU survivors and out of hospital outcomes: a cohort study. Crit Care Med 2016;44:869-79.

3 Mehlhorn J, Freytag A, Schmidt K, et al. Rehabilitation interventions for postintensive care syndrome: a systematic review. Crit Care Med 2014;42:1263-71.

4 Cuthbertson BH, Rattray J, Campbell MK, et al. The PRaCTICal study of nurse led, intensive care follow-up programmes for improving long term outcomes from critical illness: a pragmatic randomised controlled trial. BMJ 2009;339:b3723.

5 Denehy L, Skinner EH, Edbrooke L, et al. Exercise rehabilitation for patients with critical illness: a randomized controlled trial with 12 months of follow-up. Crit Care 2013;17:R156.

6 Elliott D, McKinley S, Alison JA, et al. Health-related quality of life and physical recovery after a critical illness: a multi-centre randomised controlled trial of a home-based physical rehabilitation program. Crit Care 2011;15:R142.

7 Moss M, Nordon-Craft A, Malone D, et al. A randomized trial of an intensive physical therapy program for patients with acute respiratory failure. Am J Respir Crit Care Med 2016;193:1101-10.

8 Walsh TS, Salisbury LG, Merriweather JL, et al. Increased hospital-based physical rehabilitation and information provision after intensive care unit discharge: the RECOVER randomized clinical trial. JAMA Intern Med 2015;175:901-10.

9 Griffith DM, Lewis S, Rossi AG, et al. Systematic inflammation after critical illness: relationship with physical recovery, and exploration of potential mechanisms. Thorax 2016;71:820-9.

10 Griffith DM, Vale ME, Campbell C, et al. Persistent inflammation and recovery after intensive care: a systematic review. J Crit Care 2016;33:192-9.

11 Biolo G, Fleming RY, Maggi SP, et al. Inverse regulation of protein turnover and amino acid transport in skeletal muscle of hypercatabolic patients. J Clin Endocrinol Metab 2002;87:3378-84.

12 Vesali RF, Cibicek N, Jakobsson T, et al. Protein metabolism in leg muscle following an endotoxin injection in healthy volunteers. Clin Sci 2009;118:421-7.

13 Murton AJ, Alamdari N, Gardiner SM, et al. Effects of endotoxaemia on protein metabolism in rat fast-twitch skeletal muscle and myocardium. PLoS ONE 2009;4:e6945.

14 Wilkes EA, Selby AL, Atherton PJ, et al. Blunting of insulin inhibition of proteolysis in legs of older subjects may contribute to age-related sarcopenia. Am J Clin Nutr 2009;90:1343-50.

15 Puthucheary ZA, Phadke R, Rawal J, et al. Qualitative ultrasound in acute critical illness muscle wasting. Critical Care Med 2015;43: 1603-11.
16 Tidball JG. Inflammatory processes in muscle injury and repair. Am J Physiol Regul Integr Comp Physiol 2005;288:R345-53.

17 Dos Santos C, Hussain SN, Mathur S, et al. Mechanisms of chronic muscle wasting and dysfunction after an intensive care unit stay: a pilot study. Am I Respir Crit Care Med. Published Online First: 8 Apr 2016. doi:10.1164/rccm.201512-23440C

18 Pedersen BK. IL-6 signalling in exercise and disease. Biochem Soc Trans 2007;35(Pt 5):1295-7.

19 Weber-Carstens S, Schneider J, Wollersheim T, et al. Critical illness myopathy and GLUT4: significance of insulin and muscle contraction. Am J Respir Crit Care Med 2013;187:387-96.

20 Brealey D, Brand M, Hargreaves I, et al. Association between mitochondrial dysfunction and severity and outcome of septic shock. Lancet 2002;360:219-23.

21 Puthucheary ZA, Rawal J, McPhail M, et al. Acute skeletal muscle wasting in critical illness. JAMA 2013;310:1591-600

22 Puthucheary Z, Hart N. Intensive care unit acquired muscle weakness: when should we consider rehabilitation? Crit Care 2009;13:167.

23 Chan G, Bivins-Smith ER, Smith MS, et al. Transcriptome analysis reveals human cytomegalovirus reprograms monocyte differentiation toward an M1 macrophage. J Immunol 2008;181:698-711.

24 McNelly AS, Rawal J, Shrikrishna D, et al. An exploratory study of long-term outcome measures in critical illness survivors: construct validity of physical activity, frailty, and health-related quality of life measures. Crit Care Med 2016;44:e362-9.

25 Puthucheary ZA, Denehy L. Exercise interventions in critical illness survivors: understanding inclusion and stratification criteria. Am J Respir Crit Care Med 2015;191:1464-7.

26 Herridge MS, Chu LM, Matte A, et al. The RECOVER Program: Disability risk groups \& one year outcome after $\geq 7$ days of mechanical ventilation. Am J Respir Crit Care Med. Published Online First: 14 Mar 2016. doi:10.1164/rccm.201512-23430C 